\title{
MedienPädagogik
}

Zeitschrift für Theorie und Praxis der Medienbildung

Jahrbuch Medienpädagogik 2.

Zweitveröffentlichung aus: Jahrbuch Medienpädagogik 2. (2001) Opladen: Leske + Budrich. Herausgegeben von Ben Bachmair, Dieter Spanhel und Claudia de Witt.

\section{Thesen zur medienpädagogischen Ausbildung in der Sozialpädagogik}

\author{
Heinz Moser
}

Der Diskurs über die Medien ist in den letzten 20 Jahren durch eine Verschiebung gekennzeichnet: War bisher das Fernsehen eindeutiges Leitmedium medienpädagogischer Überlegungen, so tritt gegenwärtig immer stärker der Computer in den Mittelpunkt. Damit verändert sich eine zentrale medienpädagogische Perspektive: Ging es beim Fernsehen wesentlich um Freizeit- und Unterhaltungsbedürfnisse, welche mit Hilfe eines Mediums realisiert wurden (bzw. noch werden), so ist der Computer viel stärker ein Bildungsmedium. Zwar gab es auch in diesem Bereich von allem Anfang an die Diskussion um die Videospiele; diese tritt indessen zugunsten neuer Fragestellungen zurück, die sich im Umkreis des Lernens ansiedeln: Fragen, wie diejenige nach dem Einsatz von Lernsoftware in Schule und Freizeit, nach den Möglichkeiten des virtuellen Lernens, nach dem Einbezug des Internets in Lernumgebungen und Lernwelten, prägen gegenwärtig die pädagogische Diskussion um das Medium Computer.

Aus pädagogischer Warte bedeutet dies, daß nicht die Informatik bzw. die Anwendung der Computertechnik im Zentrum informationstheoretischer Überlegungen zur Medienbildung steht, sondern die Frage, wie der Computer als Bildungsmedium und alltägliches Werkzeug im Leben der Heranwachsenden Platz finden kann bzw. muß. Die Frage des Mediums Computer bzw. der elektronischen Medien generell wird damit ein konstitutiver Teil der Reflexion auf Allgemeinbildung, oder mit anderen Worten: Die Nutzung dieser Geräte gehört für Kinder und Jugendliche zu den allgemeinen Kulturtechniken im Rahmen der menschlichen Grundbildung. In einem ähnlichen Sinn betont das BLK-Gutachten zum Einbezug der Medien sowie der Informations- und Kommunikationstechnologien in Lehr- und Lernprozesse: „Neben den traditionellen Kulturtechniken werden Fähigkeiten und Fertigkeiten im praktischen Umgang mit den neuen Informations- und Kommunikationstechnologien immer wichtiger und avancieren damit zu einer neuen Kulturtechnik. Seit den achtziger Jahren wird gefordert, die ,informationstechnische Bildung’ zu einem Bestandteil der Allgemeinbildung zu machen“ (Mandl u.a. 1998, S. 9). 
Wenn wir in diesem Zusammenhang von medienpädagogischen Aufgaben sprechen, so geht es allerdings keineswegs ausschließlich um den Computer. Vielmehr steht dieser für eine Entwicklung, welche auch die traditionellen Medien wie Radio und Fernsehen bis zu Internet und World Wide Web und andere neuartige elektronische Medien erfaßt hat. Der Schlüsselbegriff in diesem Zusammenhang ist jener der Digitalisierung. Denn seit einiger Zeit deutet sich hier eine große Fusion an: Digitalisiertes Fernsehen, Video, Computer und neuerdings auch DVD schließen sich zunehmend zusammen und gehen neue Beziehungen ein. Damit werden sich neue Anforderungen an die Medienkompetenz sowie neue medienpädagogische Fragestellungen ergeben, die den Mediengebrauch in einen übergreifenden Zusammenhang stellen, der Beruf, Freizeit und Unterhaltung gleichermaßen betrifft.

Dennoch bedeutet die beschriebene Situation nicht, daß die Inhalte der traditionellen Medienpädagogik dadurch vollständig überholt wären. Ein Anschluß an diese ergibt sich insofern, als auch die neuen elektronischen Medien stark durch eine visuelle Ansprache der Rezipienten geprägt sind. Das Verständnis von Bildsprache und ikonischen Elementen, wie es im Rahmen der Forschungen zum Fernsehen entwickelt wurde, behält auch für den Hypertext des Internets und die grafischen Präsentationsformen des WorldWideWeb seine Bedeutung. In diesem Sinne gehört ein erweiterter Textbegriff auch zukünftig zu den „fundamentals“ der Medienpädagogik. In der Verknüpfung von semiologischen und kulturellen Komponenten, wie sie in den „British Cultural Studies“ (Fiske 1989) vordemonstriert wurden, besteht die Möglichkeit, Rezeptionsweisen populär-kultureller Ereignisse durch Kinder und Jugendliche als Ausdruck einer eigenwilligen Aneignung von Medienereignissen zu verstehen.

\section{Medien in der Sozialpädagogik}

Wenn die elektronischen Medien heute zur allgemeinen Bildung und zu den alltäglichen Kulturtechniken gehören, so macht dies nicht zuletzt deutlich, daß Medienkompetenzen heute für alle Menschen wichtig sind. Im Gegensatz dazu bedeutet dies für bildungsfeme Schichten und Randgruppen, für Behinderte und für Menschen mit Sozialisationsschwierigkeiten eine zusätzliche Benachteiligung. So werden Bildungs- und Karrierechancen benachteiligter Bevölkerungsgruppen und ihre gesellschaftliche Teilhabe eingeschränkt, wenn z.B. Computerkenntnisse heute fast bei jedem Beruf vorausgesetzt werden. Aber auch Kommunikationschancen verringern sich, wenn sich die Menschen zunehmend über Internet und WorldWideWeb miteinander unterhalten.

Dies hat insbesondere die Schulen zu weitreichenden Anstrengungen veranlaßt: Die Einführung des Computers in der Volksschule sowie Projektideen wie „Schulen ans Netz" betonen die Bedeutung dieses neuen Mediums für die Chancengleichheit und das Lernen. Man könnte sich deshalb fragen, 
welches auf diesem Hintergrund noch die Aufgaben der Sozialpädagogik sind, welche diese innerhalb der Medienbildung zu übernehmen hätte. Braucht es überhaupt noch außerschulische Anstrengungen, wenn sich das öffentliche Bildungswesen dieser Fragen annimmt und die Schulen das Feld immer stärker besetzen? M.E. gibt es jedoch drei Argumentationslinien, welche aufzeigen, daß die Schulen bei der Vermittlung von Medienkompetenzen auf Grenzen stoßen:

Durch den Einbezug des Computers in die Schulen ergibt sich eine Didaktisierung und Pädagogisierung dieses Mediums. Was ich damit meine, läßt sich sehr gut am Beispiel der Lernsoftware aufzeigen. Erfahrungen zeigen nämlich, daß die bevorzugten Programme im Schul- und im Home-Bereich nicht dieselben sind. Bei einer Evaluation von Lernsoftware am Pestalozzianum Zürich ist deutlich geworden, daß sinnvolle Programme für den Unterricht einen engen Lehrplanbezug aufweisen müssen, und sie müssen in relativ überschaubaren und zeitlich gut abgrenzbaren Sequenzen zu bearbeiten sein. Befragungen von Kindern belegen demgegenüber, daß sie solche Programme als „zu stark Schule“ betrachten. Zuhause möchten sie lieber mit spielerisch angelegten Programm arbeiten, die z.B. als Abenteuer- Spiel gestaltet sind und Aspekte des Edutainment betonen. Auf dieselbe Problematik weist auch hin, daß der bekannte Software-Ratgeber von Feibel (1999) Programme häufig niedrig bewertet, welche von den Lehrern und Lehrerinnen in der genannten Software-Evaluation des Pestalozzianums Zürich als besonders brauchbar für den Unterricht befunden wurden (vgl. die Ergebnisse der Lernsoftware-Evaluation unter http://www.pestalozzianum.ch). Daraus läßt sich schließen, daß es Bereiche einer Medienkompetenz gibt, die von der Schule nur schlecht abzudecken sind. Wenn es etwa darum geht, Kriterien für „gute“ Spiele zu vermitteln oder für die kreative Arbeit an Web-Projekten zu motivieren, sind dazu - mindestens für bestimmte Gruppen von Kindern und Jugendlichen - Institutionen der außerschulischen Pädagogik besser geeignet.

Es ist gegenwärtig noch nicht so, daß schon alle Schulen über Computer verfügen. Wenn aber Chancengleichheit innerhalb nützlicher Frist realisiert werden soll, so wäre es wichtig, daß gerade die außerschulische Jugendarbeit, welche ja nicht zuletzt mit Kindern und Jugendlichen aus Randgruppen und sozial benachteiligten Schichten arbeitet, zu einem Schwerpunkt „unversehrter“ Medienarbeit würde. Ein Beispiel in diese Richtung ist etwa das Webmobil in Nordrhein-Westfalen, dessen Einrichtung Baacke wie folgt begründet: „Aber: das Lernen an der Schule erreicht nur bestimmte Schulen und Schultypen; es ist auf kognitive Orientierung ausgerichtet; viele soziale Gruppen, vor allem Mädchen, stehen weiter mehr oder weniger am Rande; die außerschulischen Erfahrungsbereiche von Jugendlichen werden kaum berührt; und schließlich: es hat sich gezeigt, daß der Anschluß von Schulen ans Netz nicht automatisch alle an kommunikative Zentren beteiligt, sondern vor allem Gebiete im ländlichen Raum oder dort, wo Metropolen fern sind, benachteiligt werden“ (Baacke 1998). 
Computer können auch dort ein wichtiges Lernmittel darstellen, wo die Sozialpädagogik in einem stärker geschlossenen Rahmen arbeitet. Denn überall dort, wo es heute um zukünftige Berufsperspektiven geht, sind Anstrengungen im Sinne einer informationstechnischen Grundbildung notwendig, Für sozial unangepaßte Jugendliche oder für behinderte Kinder und Erwachsene kann es „überlebenswichtig“ sein, in diesem Bereich Kompetenzen zu erwerben. Dies betrifft nicht zuletzt die tutoriellen Funktionen des Computers. So betont Pammer (1995, S. 12) in diesem Zusammenhang die guten Erfahrungen mit Förderungsprogrammen bei Teilleistungsschwächen. Und auch generell wird in der Literatur darauf hingewiesen, daß sich Kinder am Computer konzentrieren können, denen dies sonst schwer fällt.

Es wird deshalb notwendig sein, daß Heime nicht nur in ihrer Verwaltung Computer einsetzen, sondern diese auch ihren Klienten zugänglich machen. Ein Beispiel, wie solche Geräte vielfältig im Alltag sozialpädagogischer Institutionen eingesetzt werden können, ist die soziale Dorfgemeinschaft Herzogsägmühle - eine Einrichtung, die auf dem Internet mit Homepage und Internet-Cafe präsent ist. In ihrer Online-Zeitschrift hält sie fest: „Dabei geht es auch darum, die neuen Kommunikations- und Informationstechnologien (z.B. Email, Internet, Intranet) in die individuelle Berufsförderung einzubeziehen. Allen einen ungehinderten Zugang zu den ,Datenautobahnen’ zu ermöglichen durch besondere Massnahmen, ist seit einiger Zeit das Bemühen in Herzogsägmühle, um die Ausgrenzung benachteiligter Mitbürgerinnen und Mitbürger bei der Entwicklung der .Industriegesellschaft“ zur ,Informationsgesellschaft’ zu verhindern.“

\section{Thesen}

In Ergänzung zu diesen grundsätzlichen Überlegungen zum Medieneinsatz in sozialpädagogischen Arbeitsfeldern werden im zweiten Teil folgende Thesen zur medienpädagogischen Ausbildung in der Sozialpädagogik formuliert:

1) Die Medienpädagogik gehört zu jenen Ausbildungsgegenständen, die im Programm der sozialpädagogischen Grundausbildung verstärkt zu verankern sind. Denn Medieneinflüsse gehören heute zu den prägenden Sozialisationsbedingungen aller Heranwachsenden, und auch Kommunikations- und Lernprozesse laufen in der Informationsgesellschaft zunehmend mediatisiert ab. Das heißt u.a.:

- Unter Förderungsaspekten kann die Medienpädagogik auf neue Wege eines Enrichments durch den Einsatz von Medien hinweisen.

- Sie ermöglicht die Reflexion der charakteristischen Merkmale der Informationsgesesellschaft, die für Heranwachsende das gleichzeitige Leben in „virtuellen“ und „realen“ Welten erfordert.

- Sie kann zudem zukünftigen Sozialpädagoginnen und Sozialpädagogen zu einem vertieften Verständnis der populär-kulturellen Ausdrucksfor- 
men von Kindern und Jugendlichen helfen, die heute meist auch medial geprägt sind.

- Angesichts des vielerorts noch wenig gefestigten Bewußtseins für die hier skizzierten Fragestellungen hätte sie an exemplarischen Beispielen auf sozialpädagogische Projekte hinzuweisen, welche die elektronischen Medien in exemplarischer Weise nutzen.

2) Didaktisch wäre darauf zu achten, daß Theorie und Praxis eng miteinander verknüpft bleiben. Hier kann auf Erfahrungen der traditionellen Medienpädagogik hingewiesen werden. Danach ist es generell schwierig, Medienkompetenz allein aus theoretischer Reflexion heraus zu entwickeln. Vielmehr betonten handlungstheroretische Ansätze schon immer die Wichtigkeit der Verknüpfung mit konkreter und praxisbezogener Medienarbeit.

3) Günstig erscheint in diesem Zusammenhang eine Verbindung zu medienpädagogischer Projektarbeit, welche einen möglichst unmittelbaren Transfer zu Aufgaben zuläßt, welche sich in späteren realen Situationen der beruflichen Praxis ähnlich stellen werden. Wie man schon in der traditionellen Medienpädagogik betonte, daß ein Verständnis für die spezifischen Produktionsbedingungen von Fernsehsendungen am besten durch eigene Videopraxis zustande kommt, sollte auch die Erweiterung der Medienkompetenz im Bereich des Computers projektorientiert gestaltet werden. Im eigenen Umgang mit Medien können Studierende damit erfahren, was nach Baacke (1997, S. 56) das Zentrum einer handlungsorientierten Medienpädagogik darstellt: nämlich von der Rezeptionsorientierung zu einer aktiven Medienpartizipation zu gelangen.

4) Wesentliche Inhalte für Ausbildungselemente im Bereich der neuen Medien wären z.B.:

- das Verhältnis von Lernen und Computern (z.B. im Bereich von Lern- und StandardSoftware),

- die Frage nach dem Zusammenhang von „virtuellen“ und „realen“ Welten,

- der Bezug zu den populär-kulturellen Inhalten einer medial geprägten Alltagskultur von Kindern und Jugendlichen,

- die Bedeutung der Medien für die zukünftige Arbeitswelt,

- die gestalterischen Möglichkeiten, die durch die Fusion von früher voneinander unabhängigen Medien im Rahmen der Digitalisierung gegeben sind,

- die Veränderungen der Kommunikationsformen durch das Internet und andere globale Kommunikationsmöglichkeiten,

- der Einsatz der elektronischen Medien für Recherchen und die Informationsgewinnung.

Damit soll kein grundsätzlicher Kanon für die Ausbildung festgeschrieben werden; vielmehr sollen diese Inhalte beispielhaft verdeutlichen, wie vielfältig und von hoher lebenspraktischer Relevanz die Lerngegenstände in diesem Gebiet sind. 


\section{Literatur}

Baacke, D.: Mobile medienpädagogische Arbeit in Nordrhein-Westfalen (Download auf http://www. medienmobil.de/mobil/vortragbaacke.html)

Ders.: Medienpädagogik. Tübingen 1997

Felbel, Th.: Großer Kindersoftware-Ratgeber 1999. München 1998

Fiske, J.: Understanding Populär Culture. London 1989

Mandl, H./Reinmann-Rothmeier, G./Gräsel, C.: Gutachten zur Vorbereitung des Programms „Systematische Einbeziehung von Medien, Informations- und Kommunikationstechnologien in Lehrund Lernprozesse“. Heft 66 der BLK-Materialien zur Bildungsplanung und Forschungsförderung, Bonn 1998

Pammer, E.: Die Computerrevolution ist schon vorbei - Haben Sie (schon) gewonnen? In: Bonfranch, R. (Hrsg.): Wir können mehr als nur Schrauben verpacken ... Bern 1995 